\section{The Learning Curves for Multigenerational Transfemoral Transcatheter Aortic Valve Replacement-A Real World Analysis}

\section{Abstract}

Background: There will be an increasing demand for transcatheter aortic valve replacement (TAVR) in the years to come. Defining the learning curve for this procedure carries implications for centers looking to begin TAVR programs. There has yet to be a single center non-PARTNER-I trial TAVR learning curve in the literature. The purpose of this study is to elucidate the efficiency and mastery of one heart team at a single non-PARTNER-I trial institution using consecutive generations of TAVR devices.

Methods: In a retrospective, observational, study from 2012-2017, we reviewed data on 920 patients who underwent TAVR at our institution. Excluded were the self-expanding TAVR valves and non-transfemoral access TAVRs. We used three complementary methods (CUSUM analysis, penalized B-splines regression, and generalized linear model) on the remaining 616 patients to define the learning curve for the first 100 consecutive patients using the first (Cohort 1), second (Cohort 2) and third (Cohort 3) generation balloon-expandable TAVR valves.

Findings: In Cohort 1, the median procedure time was 178.5 minutes. Efficiency was achieved at case 40 and mastery at case 60 . The first 100 consecutive patients in Cohort 2 had a median procedure time of 73 minutes. The first 100 consecutive patients in Cohort 3 had a median procedure time of 92 minutes. There was no additional level of efficiency or mastery after the first generation TAVR valves.

Conclusion: In conclusion, given the growing number of eligible patients and expanding indications for TAVR, there will be an increased demand for TAVR. Early experience using the first generation TAVR device carried over to subsequent generations of TAVR devices.

Keywords: Aortic stenosis; Transcatheter Aortic Valve Replacement (TAVR)

\section{Giorgio A. Medranda*, Richard Schwartz, Rose Calixte and Stephen J. Green}

\author{
Deparment of Cardiology, New York \\ University Winthrop Hospital, Mineola, New \\ York, USA
}

\section{*Corresponding author: \\ Giorgio A. Medranda}

” giorgio.medranda@nyulangone.org

Deparment of Cardiology, New York University Winthrop Hospital, Mineola, New York, USA.

\section{Tel: + 516-663-9696}

\section{Citation: Medranda GA, Swartz R, Calixte} R, Green SJ (2018) The Learning Curves for Multigenerational Transfemoral Transcatheter Aortic Valve Replacement-A Real World Analysis Interv Cardiol J Vol.4 No.3:6

\section{Introduction}

There is an increasing demand for new heart teams at new centers to be capable of performing transcatheter aortic valve replacement (TAVR). With the advent of TAVR in the initial PARTNER-I trial, patients previously at high risk for surgical aortic valve replacement (SAVR) now have a less invasive alternative [1]. In the United States, the prevalence of aortic stenosis (AS) in the elderly population continues to grow [2]. As the burden of AS increases, so has the number of devices the FDA has approved for
TAVR in the United States. Despite the general similarity of access, catheterization and deployment, the newer generation valve devices offer a thinner frame and lower profile catheter [3]. The results of the PARTNER-I trials have lead to the approval of several clinical trials in the United States looking to investigate additional utilities of TAVR. Defining the learning curve for this novel yet quickly evolving procedure carries significant clinical implications for physicians and centers looking to begin TAVR programs to meet this demand. The learning curve for transfemoral TAVR (TF- 
TAVR) in the PARTNER-I trial has been described in the literature [4]. This trial included more than 1,500 patients across multiple medical centers with multiple operator heart teams [4]. There has yet to be a non-PARTNER-I trial TF-TAVR learning curve for a single center heart team described in the literature. Our objective was to elucidate the efficiency and mastery of one heart team at a single non-PARTNER-I trial institution and evaluate changes with improvements in technical design. Our results might guide future efforts to initiate TAVR programs at new or non-PARTNER-I trial centers.

\section{Methodology}

In a retrospective, observational, institutional review board exempt study from January 2012 to April 2017, we reviewed data on 920 patients with severe AS who underwent TAVR at our institution. Excluded were aborted TAVRs, CoreValve (Medtronic, Minneapolis, MN) TAVRs, CoreValve Evolut R (Medtronic, Minneapolis, MN) TAVRs and TAVR in which non-transfemoral access was obtained (Figure 1). The remaining 616 patients were then divided into three cohorts. Cohort 1 included the 176 patients who underwent TF-TAVR using the first-generation balloon-expandable SAPIEN heart-valve system (Edwards Lifesciences, Irving, CA) from January 2012 to December 2014. Cohort 2 included the 100 patients who underwent TF-TAVR using the second-generation balloon-expandable SAPIEN XT heartvalve system (Edwards Lifesciences, Irving, CA) from March 2013 to July 2015. Cohort 3 included the 340 patients who underwent TF-TAVR using the third-generation balloon-expandable SAPIEN 3 heart-valve system (Edwards Lifesciences, Irving, CA) from February 2015 to April 2017. In these three Cohorts, the first 100 consecutive patients were used for the learning curve analysis.

The TAVR procedure was performed in a sterile hybrid cardiac catheterization laboratory. Vascular access was obtained via the transfemoral approach. All TAVRs were performed by the same interventional cardiologist with the assistance of a cardiothoracic surgeon. Valve sizing was dependent on operator discretion after a thorough review of pre-TAVR multi-detector computed tomography (MDCT) and patient charts.

\section{Consort Diagram}

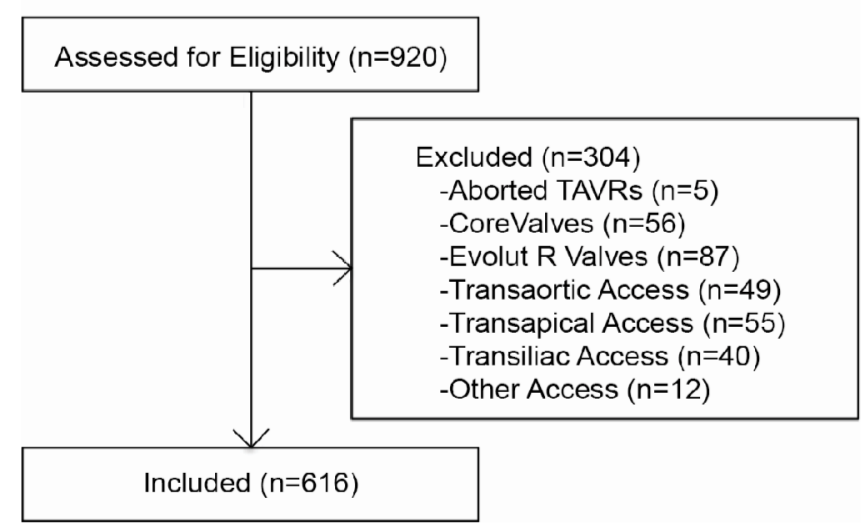

Figure 1 The consort diagram of patients in our study.
This was a study to evaluate the efficiency and mastery of TFTAVR in a new program. We analyzed the learning curve for the first 100 patients in each Cohort. We hypothesized that as the number of procedures increased with experience, the procedure time would eventually plateau. In addition, our goal was to see if there was a second or third level of mastery with regards to procedure time in the newer generation TAVR devices following experience with the first generation TAVR device. Efficiency is defined as the point on a learning curve where the operator begins to utilize procedural refinements, which lead to a gradual decrease in procedure time. Mastery is defined as the point at which the operator's procedure time becomes consistent $[5,6]$. Other procedural variables of interest were fluoroscopy time and contrast volume of each TAVR. We used three complementary methods to estimate the learning plateau: penalized basis spline (B-spline) regression, cumulative sum (CUSUM) analysis and generalized linear model (GLM).

The first method used to model the learning curve for TAVR was a penalized B-spline regression, where we use procedure number as the independent variable and searched for the best smoothing parameter lambda $(\lambda)$ [7]. B-spline regressions are a set of connected polynomial pieces used to smooth the best fitted curve due to fluctuation the outcome variables, here the time to complete the procedure, fluoroscopy time and contrast volume. In B-splines regressions, the pieces of polynomials are joined at knots. Model fits for different parameter values were compared using the Akaike information criteria (AIC) [8] and the Bayesian information criterion (BIC) to find the best fit [9] as AIC tends to overestimate while BIC tends to underestimate the dimension of a model [10].

The second method of analysis used to model the learning curve for TAVR was a CUSUM analysis [11], with median procedure time being the "predicted" value of interest to complete the procedure. We define the residual time as the difference between the actual procedure time and the median procedure time. If the operator reached mastery of the procedure, after $\mathrm{X}$ procedures, the change in the CUSUM of the residual should be negligible. We plotted the CUSUM of the difference of procedure time from the median procedure time to visually estimate $X$, the point at which the difference in the CUSUM is negligible.

Ourfinal method of analysis for the learning curve was a generalized linear model. Due to the positive skewness of procedure time, we apply a Box-Cox transformation on procedure time. Result of the box-cox transformation indicate that the best value to use was $\lambda=0$, indicating that procedure time follow a lognormal distribution [12]. That is, the natural logarithm of procedure time is normally distributed. We fit a GLM on procedure time with case number as the main predictor. Case number represents the best way to evaluate operator experience. Case number was categorized into groups of 20 consecutive patients each, with no overlap. We adjusted for age, gender, BMI, history of pacemaker, coronary artery disease with myocardial infarction, percutaneous coronary intervention, coronary artery bypass grafting, diabetes mellitus, heart failure, atrial fibrillation/flutter and access method (cutdown, percutaneous) as documented by the heart team. We used Dunnett's method of adjustment for multiple comparisons of procedure mean in each group of patients. 
Our secondary objective was to examine patient outcomes of interest, such as post-TAVR length of hospital stay, length of intensive care unit (ICU) stay and in-patient mortality before and after mastery was achieved. We compared patients' preTAVR demographic and clinical characteristics as set by the mastery cutoff point. Quantitative variables were compared using two-sample t-test and Wilcoxon rank sum test. Categorical variables were compared using the Pearson's chi-square test, with exact $p$-value computed when necessary. Comparisons of main outcomes in the full patient population between valve generations were performed using the Wilcoxon Rank sum test, with pairwise multiple comparisons done using the Dwass, Steel, and Critchlow-Flinger method. Results with $p$-value $<0.05$ were considered significant. All analyses were preformed using SAS $9.4^{\circledR}$.

\section{Results}

Of the 176 patients in Cohort 1, the first 100 consecutive patients who underwent TAVR using the first- generation balloonexpandable SAPIEN heart-valve system (Edwards Lifesciences, Irving, CA) had a mean age of $84.2 \pm 7.5$ years, with a mean BMI of $27.6 \mathrm{~kg} / \mathrm{m}^{2}$, were $52 \%$ male and $98 \%$ non-Hispanic Caucasian. These patients had a median procedure time of 178.5 minutes with an interquartile range of 156 minutes to 205.8 minutes. In Figure 2a, we present the penalized B-spline learning curve model for procedure time. Based on the B-spline regression line, the point of efficiency occurred at about case 40 and the point of mastery occurred at about case 60 . The same was found in the CUSUM analysis (Figure $\mathbf{3 a )}$ ), where the first 40 cases have procedure time larger than the median (estimated "ideal" time to complete the procedure), with fluctuations in the procedure time occurring after case 60. The GLM was adjusted for age, gender, BMI, history of pacemaker, coronary artery disease with myocardial infarction, percutaneous coronary intervention, coronary artery bypass grafting, diabetes mellitus, heart failure, atrial fibrillation/ flutter and access method (cutdown, percutaneous). Upon adjustment for patients' characteristics and procedural method, mean procedure time for group 2 (cases 21-40) was significantly higher than mean procedure time for group 5 (cases 81-100, the reference point) $(p=0.002)$. No other variables were significant in the model. In these patients, there was a 96.6\% TF-TAVR success rate prior to mastery, and a 94.9\% TF-TAVR success rate after mastery was achieved. It should be noted that although the operator switches method for the procedure from cutdown $(n=35)$ to percutaneous $(n=25)$ in the earlier cases (first 60 TFTAVR), the experienced gained still carried over to the later cases (last 40 percutaneous TF-TAVR).

When analyzing the fluoroscopy time in the first 100 patients of Cohort 1, the median was found to be 19 minutes, with an interquartile range of 14 minutes to 23 minutes. Based on the penalized B-spline analysis for fluoroscopy time, the slope was constant, indicating non-existence of a change point. The median contrast volume was 200 milliliters with an interquartile range of 140 milliliters to 260 milliliters. The B-spline analysis revealed a non-increasing and a non-decreasing slope for the regression curve, indicating no difference in contrast volume among all the cases.

With regards to the secondary outcomes of the first 100 patients in Cohort 1 , the median post-TAVR length of stay was 5 days with an interquartile range of 4 days to 7.5 days. There was no difference in post-TAVR length of stay ( 5.0 days vs. 5.5 days, $p=0.518$ ) between the two groups of patients set by the mastery cutoff point. These patients were in the ICU for a median of 30 hours with an interquartile range of 24 hours to 62 hours. There was no difference in ICU length of stay ( 28 hours vs. 34 hours, $p=0.518$ ) between the two groups of patients set by the mastery cutoff point. There was no difference for in-patient mortality (3.3\% vs. $2.5 \%, p=0.999$ ) between the two groups of patients set by the mastery cutoff point. These results are demonstrated in Table 1. When examining patient demographics between the two groups of patients set by the mastery cutoff point, there were no differences.
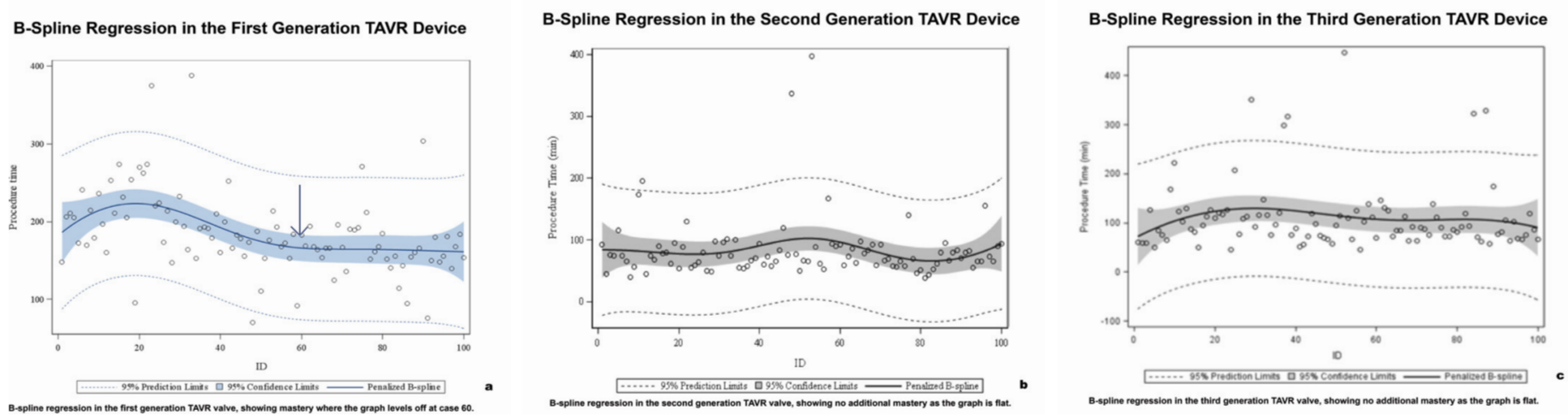

Figure 2 B-spline regression in different generations.

(a) Penalized B-spline learning curve model for procedure time in the first 100 patients using the first- generation balloon-expandable SAPIEN heart-valve system (Edwards Lifesciences, Irving, CA).

(b) Penalized B-spline learning curve model for procedure time in the first 100 patients using the second-generation balloon-expandable SAPIEN XT heart-valve system (Edwards Lifesciences, Irving, CA).

(c) Penalized B-spline learning curve model for procedure time in the first 100 patients using the third-generation balloon-expandable SAPIEN 3 heart-valve system (Edwards Lifesciences, Irving, CA). 


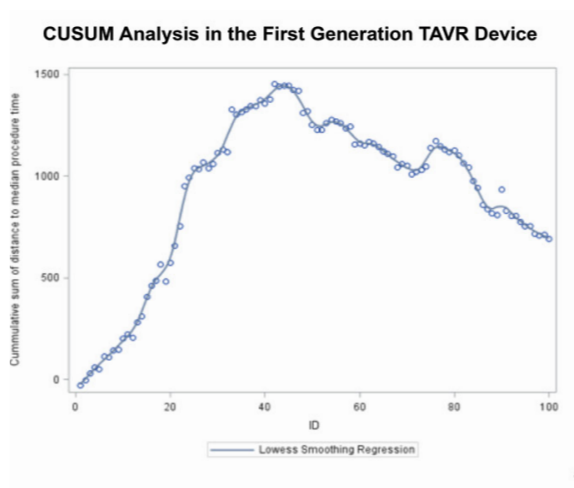

CUSUM Analysis in the Second Generation TAVR Device

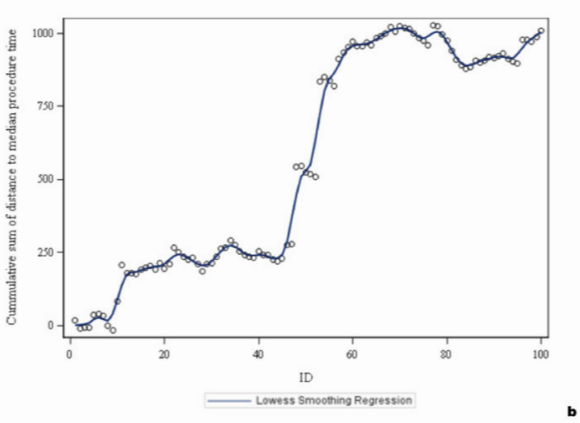

CUSUM Analysis in the Third Generation TAVR Device

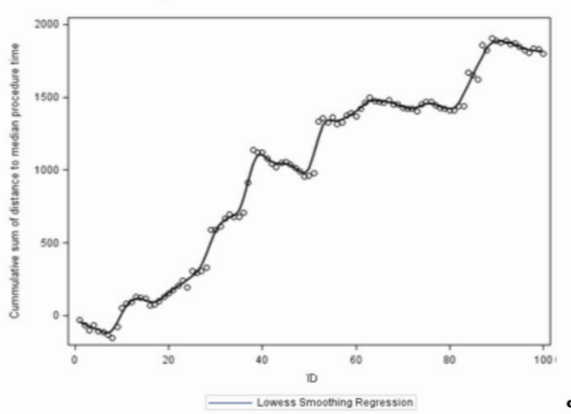

Figure 3 CUSUM analysis.

(a) CUSUM learning curve model for procedure time in the first 100 patients using the first-generation balloon-expandable SAPIEN heart-valve system (Edwards Lifesciences, Irving, CA).

(b) CUSUM learning curve model for procedure time in the first 100 patients using the second-generation balloon-expandable SAPIEN XT heart-valve system (Edwards Lifesciences, Irving, CA).

(c) CUSUM learning curve model for procedure time in the first 100 patients using the third-generation balloon-expandable SAPIEN 3 heart-valve system (Edwards Lifesciences, Irving, CA).

The 100 patients in Cohort 2 who underwent TAVR using the second-generation balloon-expandable SAPIEN XT heart-valve system (Edwards Lifesciences, Irving, CA) had a mean age of $82.71 \pm 6.96$ years, with a mean BMI of $28.5 \mathrm{~kg} / \mathrm{m}^{2}$, were $43 \%$ male and $100 \%$ non-Hispanic Caucasian. These patients had a median procedure time of 73 minutes with an interquartile range of 59 minutes to 90 minutes. The penalized B-spline regression line was relatively flat for these 100 procedures indicating no improvement from procedure 1 to procedure 100 (Figure 2b) and consistently increasing for the CUSUM model (Figure $3 \mathbf{b}$ ). This result was confirmed in the GLM model, which showed that procedure number was not associated with procedure time. Pairwise comparisons in the GLM model were not significant.

Of the 340 patients in Cohort 3, the first 100 consecutive patients who underwent TAVR using the third-generation balloonexpandable SAPIEN 3 heart-valve system (Edwards Lifesciences, Irving, CA) had a mean age of $80.58 \pm 8.61$ years, with a mean BMI of $29.5 \mathrm{~kg} / \mathrm{m}^{2}$, were $54 \%$ male and $100 \%$ non-Hispanic Caucasian. These patients had a median procedure time of 92 minutes with an interquartile range of 72 minutes to 119 minutes. The penalized B-spline regression line was relatively flat for these 100 procedures indicating no improvement from procedure 1 to procedure 100 (Figure 2c) and consistently increasing for the CUSUM model (Figure 3c). This result was confirmed in the GLM model, which showed that procedure number was not associated with procedure time. Pairwise comparisons in the GLM model were not significant.

When examining the median procedure time, we found that the first generation balloon-expandable SAPIEN heart-valve system (Edwards Lifesciences, Irving, CA) was significantly longer when compared to the median procedure times of the second generation balloon-expandable SAPIEN XT heart-valve system (Edwards Lifesciences, Irving, CA) and the third generation balloonexpandable SAPIEN 3 heart-valve system (Edwards Lifesciences, Irving, CA) (adjusted p-value<0.001). When comparing contrast volume, the third-generation valve had a lower median contrast volume than the first or second-generation valves. With regards to fluoroscopy time, the third-generation valve had a shorter median fluoroscopy time than the first or second-generation valves (adjusted $p$-value<0.001). In this third-generation valve, a level of mastery was achieved for contrast volume after case 80 and for fluoroscopy time after case 60 . This was confirmed in the CUSUM model and GLM. As the valve devices evolved, the median procedure time within each Cohort improved from 153 minutes in the first-generation device to 73 minutes in the second and third generation devices. In addition, median contrast volume showed steady improvement from 190 milliliters to 140 milliliters and finally 90 milliliters in the first, second and third generations TAVR devices respectively. The same trend was seen with median fluoroscopy time, going from 18 minutes to 11.6 and finally 7.5 minutes in the first, second and third generations TAVR devices respectively. These results are demonstrated in Table 2.

\section{Discussion}

We analyzed the TF-TAVR learning curve of one heart team at a single non-PARTNER-I trial institution using three complimentary statistical methods across three generations of TAVR devices. In the first-generation balloon-expandable SAPIEN heart-valve system (Edwards Lifesciences, Irving, CA) TF-TAVRs (Cohort 1), using penalized B-spline regression, CUSUM analysis and GLM, we found that efficiency was achieved after 40 procedures and mastery after 60 procedures. After adjusting the GLM for patients' characteristics and procedural method, mean procedure time for group 2 (cases 21-40) was significantly higher than mean procedure time for group 5 (cases $81-100$, the reference point) $(p=0.002)$. Interestingly, we found that although the operators switched methods from cutdown to percutaneous early on, the experienced gained carried over to the later cases.

Using penalized B-spline regression, fluoroscopy time and contrast volume remained unchanged as experience accumulated 
Table 1 Clinical characteristics and length of stay in ICU (hours), post-TAVR length of stay (days), ICU admissions and in-patient mortality did not differ between the first 60 cases and the last 40 cases, as set by the mastery cut-off point (Comparison of patients' outcomes before/after achieving mastery in the first generation TAVR.

\begin{tabular}{|c|c|c|c|c|}
\hline Variables & TAVR 01-60 & TAVR 61-100 & Total & p-value \\
\hline Number of Participants & 60 & 40 & 100 & -- \\
\hline Outcome Variables & -- & -- & -- & -- \\
\hline Procedure Time (Minutes) & $192.0(169.0-217.5)$ & $165.0(151.0-182.5)$ & $178.5(156.0-205.5)$ & $<0.001$ \\
\hline Length of stay in ICU (Hours) & $28.0(24.0-46.0)$ & $34.0(23.0-70.0)$ & $30.0(24.0-62.0)$ & 0.518 \\
\hline Post Procedure Length of Stay (Days) & $5.0(4.0-7.5)$ & $5.5(4.0-7.5)$ & $5.0(4.0-7.5)$ & 0.253 \\
\hline ICU Admission ( $\mathrm{N}, \%)$ & $59(98.3 \%)$ & $40(100.0 \%)$ & $99(99.0 \%)$ & 0.999 \\
\hline In Hospital Mortality (N, \%) & $2(3.3 \%)$ & $1(2.5 \%)$ & $3(3.0 \%)$ & 0.999 \\
\hline
\end{tabular}

Table 2 Mastery for procedure time was achieved using the first generation TAVR valve. Experience from this earlier generation TAVR valve carried over to subsequent generation TAVR devices. With respect to contrast volume and fluoroscopy time, there was a level of mastery achieved using the third generation TAVR valve (Comparison of learning curves across all generations of TAVR devices).

\begin{tabular}{|c|c|c|c|c|}
\hline Variables & First Generation & Second Generation & Third Generation & Total \\
\hline Total & 176 & 100 & 340 & 616 \\
\hline $\begin{array}{c}\text { Procedure Time } \\
\text { (minutes) }\end{array}$ & $153(84.5-191.5)$ & $73.0(59.0-90.0)$ & $73.0(62.0-90.0)$ & $79.5(64.0-118.5)$ \\
\hline $\begin{array}{c}\text { Contrast Volume } \\
\text { (milliliters) }\end{array}$ & $190.0(140.0-240.0)$ & $140.0(110.0-180.0)$ & $90.0(65.0-120.0)$ & $120.0(80.0-170.0)$ \\
\hline $\begin{array}{c}\text { Fluoroscopy Time } \\
\text { (minutes) }\end{array}$ & $18.0(13.5-25.0)$ & $11.6(8.4-15.5)$ & $7.5(5.5-10.7)$ & $10.4(6.5-15.9)$ \\
\hline
\end{tabular}

in the first-generation balloon-expandable SAPIEN heart-valve system (Edwards Lifesciences, Irving, CA) TF-TAVRs (Cohort 1). Additionally, when analyzing demographics and secondary outcomes of interest, we found that clinical characteristics, post-TAVR length of stay, ICU hours, in-patient mortality and ICU admissions did not differ between the first 60 cases and the last 40 cases, as set by the mastery cutoff point.

After mastery was achieved using the first-generation balloonexpandable SAPIEN heart-valve system (Edwards Lifesciences, Irving, CA) (Cohort 1), newer generations of TAVR devices were subsequently FDA approved. We analyzed the learning curve for the second-generation balloon-expandable SAPIEN XT heartvalve system (Edwards Lifesciences, Irving, CA) (Cohort 2) and the third-generation balloon-expandable SAPIEN 3 heart-valve system (Edwards Lifesciences, Irving, CA) (Cohort 3) with regards to procedure time. Within each Cohort ( 2 and 3 ), procedure time did not improve as case number increased. Early mastery using the first-generation device may have carried over despite valve evolution. The median procedure time, however, did improve dramatically when comparing the first generation balloonexpandable SAPIEN heart-valve system (Edwards Lifesciences, Irving, CA) (Cohort 1) to the median procedure times of the second generation balloon-expandable SAPIEN XT heart-valve system (Edwards Lifesciences, Irving, CA) (Cohort 2) and the third generation balloon-expandable SAPIEN 3 heart-valve system (Edwards Lifesciences, Irving, CA) (Cohort 3) $(p<0.001)$. This suggests that although mastery was obtained early on, with regards to procedure time, the heart team continued to refine their technique and show steady improvement as the TAVR devices have evolved. Of note, experience from the first two generations of TAVR devices did not carry over for contrast volume and fluoroscopy time. There was a level of mastery obtained in these parameters as the heart team began using the third-generation balloon-expandable SAPIEN 3 heart-valve system (Edwards Lifesciences, Irving, CA), likely owing slightly different techniques required with this newer valve. These results show the real-world learning curve for mastery of one heart team as the TAVR devices evolved at out institution.

The learning curve for patients enrolled in the multi-centered multi-operator PARTNER-I trial has been described in the literature [4]. In this study, they analyzed 1521 patients in the PARTNER-I trial and found that mastery was achieved after 22 cases, after which there was an $80 \%$ success rate. This was the point where a consistently low rate of major vascular complications and major bleeding was established. The most significant correlate of 30-day major adverse events and post-TAVR length of stay was procedure time $(p<0.0001)$. However, this was related to changes in the patients' risk profile, not the procedure time learning curve $(p=0.6)[4]$.

Our study examined the learning curve for TF-TAVR at a single non-PARTNER-I trial center performed by one heart team. In addition, our study demonstrated a $96.6 \%$ success rate prior to mastery, and a $94.9 \%$ success rate after mastery was achieved. Mastery was attained by our single heart team at case 60 . It was at this point where procedure time reached a consistently low point $(p=0.002)$. There was no difference in the patients' risk profile before and after the mastery cut point. Our study clarified the procedure time learning curve for TF-TAVR in a more consistent patient population.

This study evaluated the learning curve for TF-TAVR using the first-generation balloon-expandable SAPIEN heart-valve system (Edwards Lifesciences, Irving, CA), second generation balloonexpandable SAPIEN XT heart-valve system (Edwards Lifesciences, 
Irving, CA) and third generation balloon-expandable SAPIEN 3 heart-valve system (Edwards Lifesciences, Irving, CA). A limitation of our study was that the analysis applies only for the SAPIEN (Edwards Lifesciences, Irving, CA) valve series; the Core-Valve (Medtronic, Minneapolis, MN) was used less frequently and not included in the analysis. This learning curve was analyzed using single center data by a single interventional cardiologist. Similarly, a general limitation of our study was it's narrow ethnic and racial distribution. Nearly all of the patients in our study were nonHispanic Caucasian, and as a result this study could not evaluate the influence, if any, of ethnicity and race on the learning curve.

\section{Conclusion}

TAVR is a less invasive option for the treatment of symptomatic severe AS in patients at deemed to be at high risk for traditional SAVR [1]. Given the growing number of TAVR eligible patients and expanding indications for TAVR, there will be an increased demand for TAVR centers across the United States in the years

\section{References}

1 Smith CR, Leon MB, Mack MJ, Miller DC, Moses JW, et al. (2011) Transcatheter versus surgical aortic-valve replacement in high-risk patients. N Engl J Med 364: 2187-2198.

2 Osnabrugge RL, Mylotte D, Head SJ, Van Mieghem NM, Nkomo VT et al. (2013) Aortic stenosis in the elderly: Disease prevalence and number of candidates for transcatheter aortic valve replacement: $A$ meta-analysis and modeling study. J Am Coll Cardiol 62: 1002-1012.

3 Leon MB, Smith CR, Mack MJ, Makkar RR, Svensson LG, et al. (2016) Transcatheter or surgical aortic-valve replacement in intermediaterisk patients. N Engl J Med 374: 1609-1620.

4 Minha S, Waksman R, Satler LP, Torguson R, Alli O, et al. (2016) Learning curves for transfemoral transcatheter aortic valve replacement in the PARTNER-I trial: Success and safety. Catheter Cardiovasc Interv 87: 165-175.

5 Samadi D, Levinson A, Hakimi A, Shabsigh R, Benson MC (2007) From proficiency to expert, when does the learning curve for to come [2]. With respect to other institutions, starting a TAVR program, they should expect to achieve efficiency with regards to procedure time after 40 cases and mastery with regards to procedure time after 60 cases if they utilize the same heart team for each TAVR. There were no additional learning curves with regards to procedure time for subsequent TAVR devices. As the TAVR devices evolved, old experience with regards to procedure time was found to carry over. Interestingly, the first two generations of TAVR devices did not demonstrate learning curves for contrast volume and fluoroscopy time. There was a level of mastery obtained in contrast volume and fluoroscopy time as the heart team began using the third generation TAVR device, likely owing slightly different techniques required with this newer valve. Our analysis determined the point of efficiency and mastery of one TAVR team, at our non-PARTNER-I trial institution, which may guide future efforts of operators at other non-trial institutions as TAVR valves continue to evolve. robotic-assisted prostatectomies plateau? The Columbia University experience. World J Urol 25: 105-110.

6 Seamon LG, Fowler JM, Richardson DL, Carlson MJ, Valmadre S, et al (2009) A detailed analysis of the learning curve: robotic hysterectomy and pelvic-aortic lymphadenectomy for endometrial cancer. Gynecol Oncol 114: 162-167.

7 Eilers P, Marx BD (1996) Flexible smoothing with B-splines and penalties. Statist Sci 11: 89-121.

8 Akaike $\mathrm{H}$ (1974) A new look at the statistical model identification. IEEE Trans Automat Contr 19: 716-723.

9 Schwarz G (1978) Estimating the dimension of a model the annals of statistics. Ann Statist 6: 461-464.

10 Dziak JJ, Coffman DL, Lanza ST, Li R (2012) Sensitivity and specificity of information criteria. Peer J 1: 1-20.

11 Page ES (1954) Continuous inspection schemes. Biometrika 41: 100-115.

12 Box GEP, Cox DR (1964) An analysis of transformations. J Roy Stat Soc B Met 26: 211-252. 